DOI: $10.34185 / 1991-7848.2018 .01 .04$

УДК 621.928.024.1

Д.А. Кононов, И.В. Пелых, Ж.Б. Пинто

\title{
ГАРМОНИЧЕСКИЙ АНАЛИЗ УПРУГОГО КОЛОСНИКОВО-КАРТОЧНОГО ЭЛЕМЕНТА ДИНАМИЧЕСКИ АКТИВНОЙ ПРОСЕИВАЮЩЕЙ ПОВЕРХНОСТИ ВИБРАЦИОННОГО ГРОХОТА
}

\begin{abstract}
Поверхностные вибрирующие сита должны обеспечивать довольно сложное движение просеивания частии сыпучего материала. Для обеспечения необходимых условий для работы просеивающих поверхностей предлагается использовать новую конструкцию динамической упругой поверхностноактивной просеивающей решетки самоочищающейся решетки - карты. Композитные упругие элементы решетки-карты без предварительной деформации, установленные в коробке вибрационного грохота. Консольные горизонтальные полки решетки элементов карты под воздействием виброустойчивых данных совершают сложные вибрационно-пространственные движения. Поскольку пласт URCC апертуры «активен» консольными горизонтальными полками, возникает необходимость в изучении их траекторий движения с учетом конструктивных параметров сита и вибрационной камеры вынужденных колебаний. Цель исследования: метод определения собственных частот и форм мод упругого (резинового) элемента, образующего тканевый ситовый вибратор, который достигнет наибольшей амплитуды колебаний при работе вибрационного грохота. Задача исследования: разработать модель решетчатого элемента карточного сита URCC для гармонического анализа методом конечных элементов. Поскольку элемент решетки имеет сложную форму, предлагается решение с использованием метода конечных элементов.

Рассматривается решение задачи гармонического анализа движения составного элемента колосниково-карточного сита вибрационного грохота с помощью метода конечных элементов грохота. Для существующего элемента определено, что при диапазоне варьирования модуля упругости $E=6,3 \ldots 25 \mathrm{MПа,} \mathrm{собственная} \mathrm{частота} \mathrm{горизонтальных} \mathrm{колебаний} \mathrm{изменилась} \mathrm{в}$ пределах $17 . . .34$ Ги, а вертикальных - 37...72 Гц.
\end{abstract}

Ключевые слова: карточное сито, гармонический анализ, собственная частота, модуль упругости.

Постановка задачи. Просеивающая поверхность вибрационных грохотов должна обеспечить достаточно сложные движения отсеиваемых частиц сыпучего материала.

Во-первых, отсеиваемые частицы должны переместиться с верхнего слоя до слоя, контактирующего с просеивающей поверхностью. Во-вторых, обеспечить проход частицы через калибрующую ячейку. В-третьих, если частица застрянет в просеивающем отверстии ячейке (так называемое «трудное зерно», размеры которого незначительно больше размера ячейки) должно произойти самоочистка сита, при котором «трудное зерно» должно быть вытолкнуто из ячейки

Для обеспечения данных условий работы просеивающих поверхностей листовидного типа, предлагается использовать новую конструкцию упругой динамически активной просеивающей поверхности [1] - сита самоочищающегося колосниково - карточного (ССКК) (рис.1 а-б), составные упругие колосниково-карточные элементы которого, без предварительной деформации устанавливаются в коробе вибрационного грохота.

Основными структурными частями динамически активного сита самоочищающегося колосниково-карточного (ССКК) (рис.1-а) шихтового вибрационного грохота, являются установленные без предварительной деформации (натяжения) в пазах продольных колосникообразных опор съемные упругие колосниково-карточные элементы (ККЭ) (рис.1-б), имеющие криволинейно изогнутую рабочую поверхность переменной толщины. При этом консольные горизонтальные полки данных колосниково-карточных элементов непосредственно формируют ситовое полотно ССКК, которое под воздействием сил вибрации совершает сложные колебательно-прострнственные перемещения $[1,4,5]$.

(c) Кононов Д.А., Пелых И.В., Пинто Ж.Б., 2018 


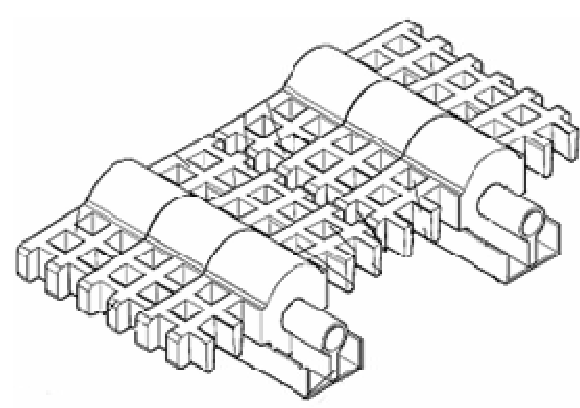

a)

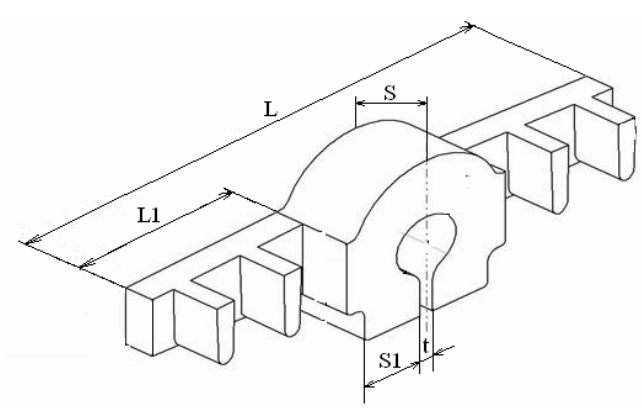

б)

Рисунок 1 - Динамически активная просеивающая поверхность щихтового вибрационного грохота - сито самоочищающееся колосниково-карточное ССКК:

а) общий вид сита ССКК; б) съемный упруго-эластичный колосниково-карточный элемент, формирующий ситовое полотно ССКК

Поскольку формирование апертуры ССКК происходит «активными» консольными горизонтальными полками, возникает необходимость в исследовании траекторий их движения с учетом конструктивных параметров сита, а также вынужденных колебаний короба вибромашины.

Разработанное ССКК обеспечивает движения частиц для этих трех случаев. За первый случай, отвечает увеличенное колебание сита в нормальном направлении, при котором увеличивается «виброкипение» слоя. За третий случай, отвечает возможные колебания колосниковых элементов в плоскости сита, при этом происходит изменения размеров ячеек в свете, что способствует самоочищению.

Несмотря на многочисленные исследования, связанные с разработкой данных разновидностей просеивающих поверхностей, вопросы их кинематики, находящиеся в зависимости от вынужденных колебаний короба вибрационного грохота, в настоящее время еще недостаточно хорошо изучены [6].

Цель исследования: предложить методику определение собственных частот и форм колебания упругого (резинового) элемента, формирующего ситовое полотно вибромашины, что позволит достигать наибольших амплитуд колебания при работе вибрационного грохота.

Задача исследования: разработать модель колосниково-карточного элемента сита ССКК для гармонического анализа с помощью метода конечных элементов.

Теоретические основы. Гармонический анализ предназначен для решения уравнений движения в случае установившихся колебательных процессов (вынужденных колебаний). Рассматривается общее уравнение движения [2, 3]

$$
[M]\{\ddot{u}\}+[C]\{\dot{u}\}+[K]\{u\}=\left\{F^{a}\right\},
$$

где $[M]$ - матрица масс; [C] - матрица демпфирования; [K] - матрица жесткости; $\{\ddot{u}\}$ - вектор ускорений в узлах; $\{\dot{u}\}$ - вектор скоростей в узлах; $\{\mathrm{u}\}$ - вектор перемещений в узлах; $\left\{F^{a}\right\}-$ вектор приложенных внешних усилий.

Предполагается, что при установившемся процессе все точки конструкции движутся с одной и той же заданной частотой, но, возможно, с различными сдвигами по фазе. Причиной возникновения сдвига по фазе может явиться наличие демпфирования. Следовательно, перемещения можно представить в виде:

$$
\{u\}=\left\{u_{\max } e^{i \varphi}\right\} e^{i \Omega t}
$$

где $u_{\text {max }}$ - амплитуда перемещений; $i$ - мнимая единица; $\Omega=2 \pi f$ - внешняя круговая частота; $f$ - частота внешних сил; $t$ - время; $\varphi$ - сдвиг фаз для перемещений (в радианах).

Заметим, что $u_{\max }$ и $\varphi$ могут быть различны для различных степеней свободы. 
Для формулировки проблемы вынужденных колебаний используются комплексные обозначения. В этом случае предыдущее уравнение можно представить в виде:

$$
\{u\}=\left\{u_{\max }(\cos \phi+i \sin \phi)\right\} e^{i \Omega t}
$$

или

$$
\{u\}=\left(\left\{u_{1}\right\}+i\left\{u_{2}\right\}\right) e^{i \Omega t}
$$

где $\left\{u_{1}\right\}=\left\{u_{\max } \cos \varphi\right\}$ - действительная часть; $\left\{u_{2}\right\}=\left\{u_{\max } \sin \varphi\right\}-$ мнимая часть вектора перемещений.

Вектор сил может быть определен аналогично вектору перемещений:

$$
\{F\}=\left\{F_{\max } e^{i \psi}\right\} e^{i \Omega t}
$$

или

$$
\{F\}=\left\{F_{\max }(\cos \psi+i \sin \psi)\right\} e^{i \Omega t}
$$

или

$$
\{F\}=\left(\left\{F_{1}\right\}+i\left\{F_{2}\right\}\right) e^{i \Omega t}
$$

где $F_{\max }$ - амплитуда сил; $\psi$ - сдвиг фаз для сил (в радианах); $\left\{F_{1}\right\}=\left\{F_{\max } \cos \psi\right\}$ действительная часть вектора сил; $\left\{F_{2}\right\}=\left\{F_{\max } \sin \psi\right\}-$ мнимая часть вектора сил.

Подставляя (9), (10) в (8), получим:

$$
\left(-\Omega^{2}[M]+i \Omega[C]+[K]\right)\left(\left\{u_{1}\right\}+i\left\{u_{2}\right\}\right) e^{i \Omega t}=\left(\left\{F_{1}\right\}+i\left\{F_{2}\right\}\right) e^{i \Omega t}
$$

Так как зависимость от времени $\left(e^{i \Omega t}\right)$ одинакова для обеих частей уравнения, то она может быть опущена:

$$
\left([K]-\Omega^{2}[M]+i \Omega[C]\right)\left(\left\{u_{1}\right\}+i\left\{u_{2}\right\}\right)=\left(\left\{F_{1}\right\}+i\left\{F_{2}\right\}\right) .
$$

Так как колосниково-карточный элемент имеет сложную форму, то предлагается для решения использовать метод конечных элементов.

Для проведения гармонического анализа при конечно-элементной постановке вопроса доступны три метода: полный, сокращенный и метод суперпозиции мод.

Процедура гармоническоо анализа состоит из четырех основных шагов: построение модели; определение типа анализа, задание опций, приложение воздействий и получение решения, «экспандирование» мод, анализ результатов.

Свойства материала должны быть линейными, изотропными или ортотропными, постоянными или зависящими от температуры. Нелинейные свойства (нелинейная упругость, пластичность, ползучесть) игнорируются.

Вследствие упрощения и сокращения времени расчёта рассмотрим половину колосниково-карточного элемента, наложив соответствующие ограничения. Общий вид объемной модели показан на рис. 2.

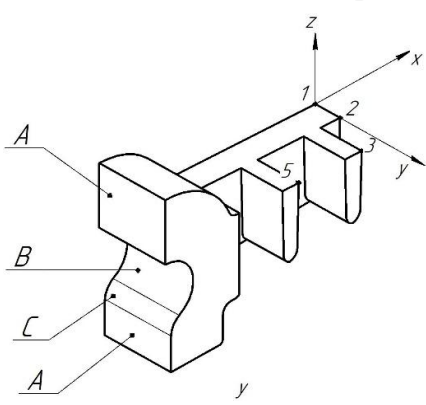

Рисунок 2 - Общий вид элемента

В соответствие с принятыми допущениями накладываются следующие граничные

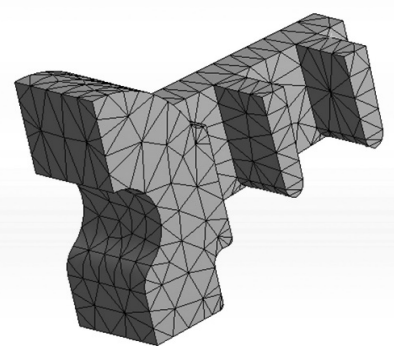

Рисунок 3 - Конечно-элементная модель условия: 
- поверхности А не могут перемещаться в нормальном направлении, $\left(\delta_{x}=0\right)$, ;

- поверхность В и С не могут перемещаться в направлении оси $y,\left(\delta_{y}=0\right)$.

В качестве базового элемента примем колосниково-карточный элемент экспериментальной модели вибрационного грохота, разработанного авторами на кафедре МАМП Национальной металлургической академии Украины $[5,6]$.

Конечно-элементная модель показана на рис. 3 (общее число узлов 3143, элементов 1595). В качестве материала используется резина 2959 со следующими характеристиками: плотность $\rho=1200 \mathrm{kz} / \mathrm{M}^{3}$; модули упругости $E=12 M \Pi a, G=4,1 M П а$; коэффициент Пуассона $\mu=0,48$.

При расчете исследованного движения характерных точек 1-5, при различном приложении к ним возбуждающих сил. Изменение частоты возмущающей силы 0 ... 120 Гц, при рабочей частоте грохота 25 Гц. На рис. 4 представлены амплитудно-частотные и фазнјчастотные характеристики для точки 1.

Как видно из графика имеются две ярко выраженные частоты для горизонтальных колебаний $f_{1}=27,2$ Гц; и вертикальных $-f_{2}=57,2$ Гц.

Для остальных рассматриваемых точек расчетные зависимости имеют аналогичный вид.

Как известно, для консольной защемленной балки значения собственных частот определяются по формуле, в Гц

$$
f^{\prime}=\frac{\alpha^{2}}{2 \pi L^{2}} \sqrt{\frac{E I}{\rho A}},
$$

где . $\alpha$. - расчетный коэффициент, для первой частоты $\alpha=1,875 ; L$ - длина балки; $A$ площадь поперечного сечения; I - момент инерции поперечного сечения.

Заменяя исследуемый элемент ССКК балкой постоянного прямоугольного сечения, получаем следующие значения собственных частот $f_{Z}^{\prime}=26,2$ Гц и $f_{y}^{\prime}=61,2$ Гц . Как видно, для горизонтальных колебаний частоты хорошо согласуются.

Частоты собственных колебаний зависят от геометрических размеров ССКК и физикомеханических свойств резины (плотность, модуль упругости и коэффициент Пуассона).

На практике наиболее просто варьировать механическими свойствами резины, т.к. стоимость пресс-формы, которая задает геометрические размеры, достаточно высока.

На рис. 5 приведены амплитудно-частотные характеристики колебаний точки 1 при различных значениях модуля упругости.

Для учета реальных свойств материала при данном расчете был введен коэффициент демпфирования. Это позволяет уменьшить амплитуды колебаний, которые при резонансной частоте теоретически стремятся к бесконечности.

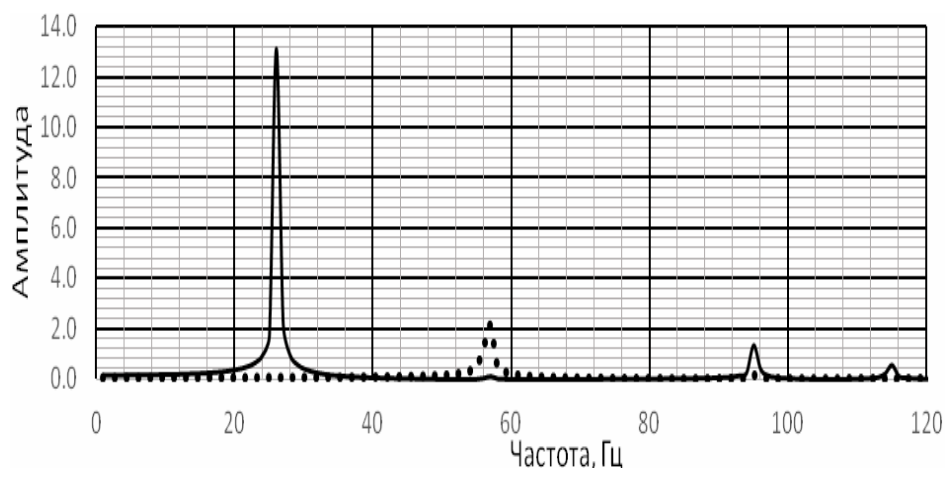

Рисунок 4 - Амплитудно-частотная характеристика карточного элемента («---»- горизонтальные колебания, «•••»-вертикальные) 


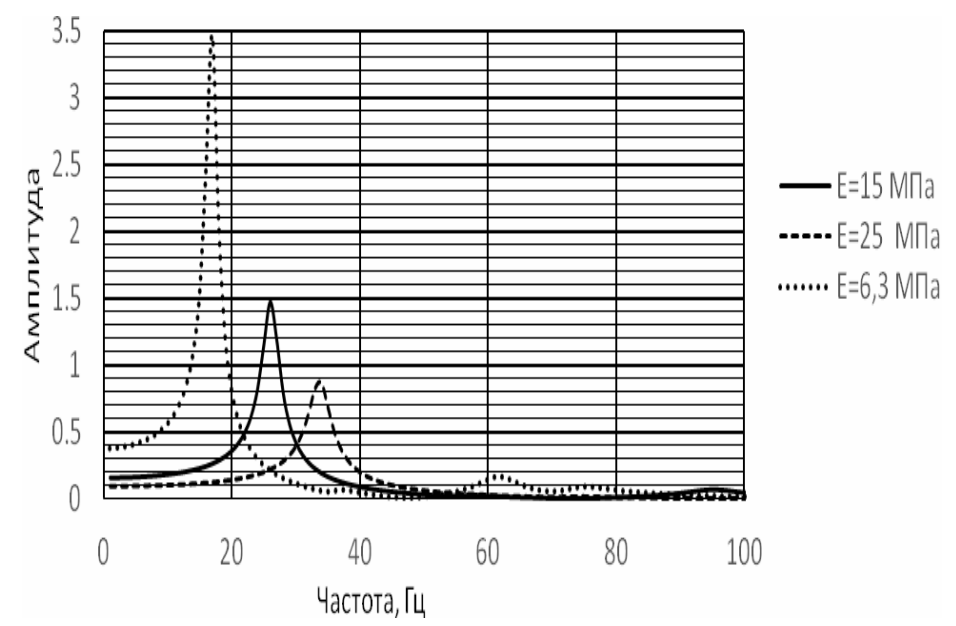

Рисунок 5 - Амплитудно-частотная характеристики карточного элемента при различных значениях модуля упругости (Е) резины

Как видно с возрастанием модуля упругости частота собственных колебаний возростает, а амплитуда уменьшаются.

На практике, при изготовлении колосниково-карточного элемента, необходимый модуль упругости можно выбирать следующим образом. Если в сортируемом сыпучем материале содержится большое количество подрешетного продукта, то необходимо интенсивно воздействовать на материал для качественной сегрегации, т.е. вертикальные составляющие колебаний должны быть максимальны. Если в исходном материале много «трудных зерен», то необходимо улучшить самоочищаемость сита, и, следовательно, увеличить колебания колосников в горизонтальной плоскости.

При диапазоне варьирования модуля упругости $E=6,3 \ldots 25 M П а$, собственные частоты горизонтальных колебаний изменились в пределах 17...34 Гц, а вертикальных - 37...72 Гц. Для более существенного диапазона изменения частот необходимо изменять форму и размеры колосниково-карточных элементов.

\section{Выводы}

В статье рассматривается решение задачи гармонического анализа движения структурного элемента разработанного в НМетАУ колосниково-карточного сита, являющегося просеивающей поверхностью вибрационного грохота, с помощью метода конечных элементов.

Для существующего элемента определено, что при диапазоне варьирования модуля упругости $E=6,3 \ldots 25 M П a$, собственная частота горизонтальных колебаний изменилась в пределах $17 . . .34$ Гц, а вертикальных - 37...72 Гц.

\section{ЛИТЕРАТУРА}

1. Патент № 103727 Україна, МПК В 07 В 1/46. Сито вібраційного грохота/ Білодіденко С.В., Пелих І.В., Кононов Д.О., Петренко В.О., Бородай В. І.; заявник і власник Національна металургійна академія України. - № А 201212361; заявл. 29.10.2012; опубл. 11.11.2013, Бюл. №21.

2. Бате К., Вилсон Е. Численные методы анализа и метод конечных элементов. М.: Стройиздат, 1982.

3. Леонтьев Н.B. Применение системы ANSYS к решению задач модального и гармонического анализа. Учеб. метод. пособие. - Н. Новгород: Изд-во ННГУ, 2006. - 101 с.

4. Рациональная конструкция просеивающей поверхности/вибрационного грохота металлургического производства/ Белодеденко С.В., Петренко В.А., Пелых И.В. //Металлургическая и горнорудная промышленность. - 2009. -№4. - с. 79 - 82.

5. Исследование процесса грохочения кокса на модели вибрационного грохота / С.В. Белодеденко, Д.А. Кононов, И.В. Пелых // Металлургическая и горнорудная промышленность. - 2013. - №4. 
6. Модальный анализ упругого колосниково - карточного элемента просеивающей поверхности шихтового вибрационного грохота/ Кононов Д.А., Пелых И.В. //Системні технології. Регіональний міжвузівський збірник наукових праць - НМетАУ, Дніпро. - 2017. вып. 2 (109).

\section{REFERENCES}

1. Patent \# 103727 UkraYina, MPK V 07 V 1/46. Sito vIbratsIynogo grohota/ BIlodIdenko S.V., Pelih I.V., Kononov D.O., Petrenko V.O., Boroday V. I.; zayavnik I vlasnik NatsIonalna metalurgIyna akademIya UkraYini. - \# A 201212361; zayavl. 29.10.2012; opubl. 11.11.2013, Byul. \#21.

2. Bate K., Vilson E. Chislennyie metodyi analiza i metod konechnyih elementov. M.: Stroyizdat, 1982.

3. Leontev N.V. Primenenie sistemyi ANSYS k resheniyu zadach modalnogo i garmonicheskogo analiza. Ucheb. metod. posobie. - N. Novgorod: Izd-vo NNGU, 2006. - $101 \mathrm{~s}$.

4. Ratsionalnaya konstruktsiya proseivayuschey poverhnosti/vibratsionnogo grohota metallurgicheskogo proizvodstva/ Belodedenko S.V., Petrenko V.A., Pelyih I.V. //Metallurgicheskaya i gornorudnaya promyishlennost. - 2009. - \#4. - s. 79 - 82.

5. Issledovanie protsessa grohocheniya koksa na modeli vibratsionnogo grohota / S.V. Belodedenko, D.A. Kononov, I.V. Pelyih // Metallurgicheskaya i gornorudnaya promyishlennost. - 2013. - \#4.

6. Modalnyiy analiz uprugogo kolosnikovo - kartochnogo elementa proseivayuschey poverhnosti shihtovogo vibratsionnogo grohota/ Kononov D.A., Pelyih I.V. //SistemnI tehnologIYi. RegIonalniy mIzhvuzIvskiy zbIrnik naukovih prats - NMetAU, DnIpro. - 2017. vyip. 2 (109).

Received 19.01.18

\section{HARMONIC ANALYSIS OF THE ELASTIC GRATE - CARD ELEMENT OF THE DYNAMICALLY ACTIVE SIEVING SURFACE OF THE VIBRATING SCREEN}

Sieve surface vibrating screens should provide fairly complex movement of the bulk material particles sifting. In order to ensure the necessary conditions for the work of screening surfaces, it is proposed to use the new design dynamic elastic surface- active screening sieves self-cleaning grate card. Composite elastic grate-card elements without preliminary deformation, installed in the box vibrating screen. Console horizontal shelves grate card elements under the influence of vibration strength data makes sophisticated vibration-spatial movement. Since the formation URCC aperture is "active" cantilevered horizontal shelves, it becomes necessary to study their motion trajectories in view of the design parameters of the sieve and forced vibrations vibrator box.

Purpose of the study: The method of determination of natural frequencies and mode shapes of the elastic (rubber) member forming fabric sieve vibrator, which will reach the greatest amplitude oscillations during operation of the vibrating screen. The research problem: develop a model gratecard element sieve URCC for harmonic analysis with the finite element method. Since the grate-card element has a complex shape, it is proposed for the solution of using the method of finite elements.

Harmonic analysis procedure consists of four main steps: model building; definition of the type of analysis, the job options, impacts the application and obtain a decision, "of expansion" mode, analysis of the results. The properties of the material should be linear and isotropic or orthotropic, constant or temperature-dependent. The nonlinear properties (nonlinear elasticity, plasticity, creep) are ignored. Due to simplify and reduce the time calcgthat considered half-card grate element, which kalozheny appropriate limits.

As the base member will take-card grate element experimental vibrating screen model developed by the authors at the Department IMMTA National Metallurgical Academy of Ukraine. In the tires used in 2959 with the following characteristics as the material: density $\rho=1200 \mathrm{kz} / \mathrm{M}^{3}$; elastic moduli $E=12 \mathrm{MPa}, G=4,1 \mathrm{MPa}$; Poisson's ratio $\mu=0,48$. Changing the frequency of the disturbing forces $0 \ldots 120 \mathrm{~Hz}$, at an operating frequency of $25 \mathrm{~Hz}$ screen. The figures show the amplitude-frequency and phase-frequency characteristics for different points. On the chart, there are two clearly defined natural frequencies for horizontal vibrations $f_{1}=27,2 \mathrm{~Hz}$; and vertical $f_{2}=57,2 \mathrm{~Hz}$. 
When replacement of an element of constant beam of rectangular cross section, we obtain the values of natural frequencies $f_{z}^{\prime}=26,2 \mathrm{~Hz}$ and $f_{y}^{\prime}=61,2 \mathrm{~Hz}$. As can be seen, for horizontal oscillation frequencies are in good agreement. Eigenfrequency depends on the geometric dimensions and physical and mechanical rubber properties (density, elastic modulus and Poisson's ratio).

In practice, the most easily varied mechanical properties of rubber, as mold value that specifies the geometric dimensions, is quite high. Graphs show the amplitude-frequency characteristics of the oscillation of points with different values of the modulus of rubber elasticity. With increase of the modulus of elasticity increases the natural frequency and the amplitude are reduced. In practice, in the manufacture of card-grate element, it is possible to select the desired modulus follows. If the sorted bulk material contains a large amount of undersize product, it is necessary to intensively act on the material quality of segregation, i.e. vertical components of oscillation should be maximized. If a lot of "hard grains" in the starting material, it is necessary to improve the self-cleaning screens, and therefore increase the grate fluctuation in the horizontal plane.

When the range of variation of the modulus of elasticity $E=6,3 \ldots 25 \mathrm{MPa}$, horizontal oscillation eigenfrequencies changed within $17 \ldots 34 \mathrm{~Hz}$, and the vertical - $37 \ldots 72 \mathrm{~Hz}$. For more substantial frequency range necessary to change the shape and dimensions of the card-grate elements.

Keywords: card sieve, harmonic analysis, natural frequency, modulus of elasticity.

\section{ГАРМОНІЙНИЙ АНАЛІЗ ПРУЖНОГО КОЛОСНИКОВИХ - КАРТКОВОГО ЕЛЕМЕНТА ДИНАМІЧНО АКТИВНОЇ ПОВЕРХНІ, ЩО ПРОСІЮЄ ВІБРАЦІЙНОГО ГУРКОТУ}

Розглядається рішення задачі гармонійного аналізу руху складового елементу Колосникове-карткового сита вібраційного гуркоту за допомогою методу скінченних елементів. гуркоту, за допомогою методу скінченних елементів. Для існуючого елемента Колосникове-карткового сита визначено, що при діапазоні варіювання модуля пружності гуми $\mathrm{E}=6,3 \ldots 25$ МПа, власна частота горизонтальних коливань змінюється в межах $17 \ldots 34$ Гц, а вертикальних - 37 ... 72 Гц.

Ключові слова: карткове сито, гармонійний аналіз, власна частота, модуль пружності.

Кононов Дмитрий Александрович - к.т.н., доцент, кафедра машин и агрегатов металлургического производства, Национальная металлургическая академия Украины.

Kononov Dmitriy - Ph.D., Associate Professor, Department of Machines and Units of Metallurgical Production, National Metallurgical Academy of Ukraine.

Пелых Игорь Владимирович - к.т.н., ведущий специалист, ЧАО «Евраз -Днепровский металлургический завод».

Pelykh Igor - Ph.D., Leading Specialist, PJSC “Evraz-Dneprovsky Metallurgical Works”.

Пинто Жозе Брони - студент, Национальная металлургическая академия Украины.

Pinto Jose Broni - student, National Metallurgical Academy of Ukraine. 